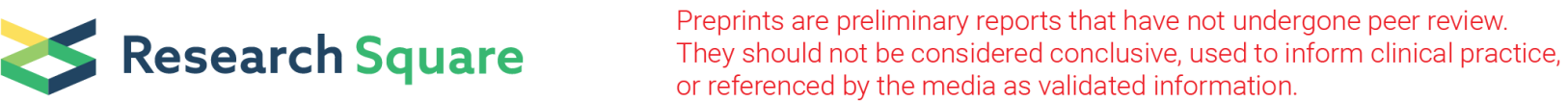

\section{White Matter Damage as a Consequence of Vascular Dysfunction in a Spontaneous Mouse Model of Chronic Hypoperfusion with eNOS Deficiency}

\section{Xingyong Chen}

Fujian Institute of Medical Sciences

\section{Ling Chen}

The school of basic medical sciences

\section{Geng Lin}

University Tennessee Health Science Center

Mahesh Chandra Kodali

UTHSC: The University of Tennessee Health Science Center

\section{Mingqi Li}

The University of Tennessee Health Science Center

\section{Sarah Grace Lebovitz}

The University of Tennessee Health Science Center

Tyler C. Ortyl

The University of Tennessee Health Science Center

\section{LeXiao Li}

The University of Tennessee Health Science Center

\section{Saifudeen Ismael}

The University of Tennessee Health Science Center

\section{Purnima Singh}

The University of Tennessee Health Science Center

\section{Kafait U Malik}

The University of Tennessee Health Science Center

\section{Tauheed G Ishrat}

The University of Tennessee Health Science Center

\section{Wei Zheng}

Basic medical university

Francesca-Fang Liao ( $\nabla$ fliao@uthsc.edu )

University of Texas Health Science Center at Houston https://orcid.org/0000-0001-5144-3600 


\section{Research article}

Keywords: Vascular cognitive impairment and dementia, Alzheimer's disease, Endothelial nitric oxide synthase, Chronic cerebral hypoperfusion, Demyelination, Axonal dysfunction, Astrogliosis, White matter inflammation, Blood brain-barrier leakage, Nitric oxide signaling.

Posted Date: June 7th, 2021

DOl: https://doi.org/10.21203/rs.3.rs-563166/v1

License: (c) (i) This work is licensed under a Creative Commons Attribution 4.0 International License. Read Full License 


\section{Abstract}

Background: Vascular cognitive impairment and dementia (VCID) is the second most common form of dementia after Alzheimer's disease (AD). Currently, the mechanistic insights into the evolution and progression of VCID are not fully understood. White matter change represents an invariant feature of both VCID and AD. Compelling clinical neuroimaging and pathological evidence suggest a link between white matter changes and neurodegeneration. Our prior study detected non-perfusion lesions in mice with partial deficiency of endothelial nitric oxide (eNOS) expression at a very young age. These lesions developed in multiple brain regions in an age-dependent manner, precisely matching to those hypoperfused areas identified in preclinical $A D$ patients (i.e., temporoparietal and retrosplenial granular cortexes, and hippocampus). We therefore reasoned that eNOS- deficient mice could serve as a spontaneous model of chronic hypoperfusion.

Methods/Results: White matter tracts are particularly susceptible to the vascular damage induced by chronic hypoperfusion. Using immunohistochemistry, we detected massive demyelination in the middle aged eNOS-deficient mice. The demyelinated areas were confined to cortical and subcortical areas including the corpus callosum and hippocampus, but did not involve the striatum. The intensity of demyelination correlated with behavioral gait deficits. By Evans blue angiography, we detected bloodbrain barrier (BBB) leakage as another early pathological change affecting frontal and parietal cortex in eNOS-deficient mice, which occurs in as early as 3-4 months of age. Sodium nitrate fortified drinking water provided to young and middle aged eNOS-deficient mice completely prevented non-perfusion, BBB leakage, and white matter pathology, indicating that impaired endothelium-derived NO signaling may have caused these pathological events.

Conclusions: Using eNOS-deficient mice, we identified BBB breakdown and non-perfusion as the two earliest pathological events, resulting from insufficient vascular NO signaling. We speculate that the compromised BBB and chronic hypoperfusion trigger vascular damage, along with oxidative stress and astrogliosis, accounting for the white matter pathological changes in the eNOS-deficient mouse model. We conclude that eNOS-deficient mice represent an ideal spontaneous evolving model for studying the earliest events leading to spontaneous white matter changes, which will be instrumental to future therapeutic testing of drug candidates and for targeting novel/specific vascular mechanisms contributing to $V C I D$ and $A D$.

\section{Background}

Vascular cognitive impairment and dementia (VCID), is the second most common form of dementia in the elderly after Alzheimer's disease (AD) [1] and is caused by insufficient blood supply to the brain. The prevalence of mixed dementia with clinical and pathological features of both VCID and AD is increasing; cerebrovascular pathology is present in at least half of all dementia cases [2-4]. VCID encompasses a broad spectrum of cerebrovascular-driven cognitive impairment, from mild cognitive impairment to fully developed dementia. This disease state is often further complicated by genetic factors (ApoE4), 
metabolic disorders (hypertension, type 2 diabetes, high cholesterol, hyperhomocysteinemia) and lifestyle factors (smoking, inactivity, excessive alcohol consumption) [5-7]. These risk factors often result in chronic cerebral hypoperfusion and microvascular pathology [8-11]. They are the most common pathologies that co-occurs in VCID and AD $[12,13]$. Although there are several good models to stimulate individual aspects of VCID [e.g., in vitro models of the neurovascular units, surgical models of chronic cerebral hypoperfusion induced by bilateral common carotid artery stenosis (BCAS), animals with NOTCH3 mutations as a model of small vessel disease], the heterogeneity of the disease states limits any of them from being a perfect model of all aspects of the disease $[14,15]$. Therefore, there is a pressing need to develop representative animal models to better understand the underlying mechanisms and identify preclinical biomarkers, prevention and treatment strategies.

Our prior work based on mice partially deficient in expressing endothelial nitric oxide (eNOS) gene has identified a potentially excellent model for studying the contribution of vascular dysfunction to dementia [16]. Endothelial eNOS-deficient mice spontaneously develop multiple hypoperfused/occluded areas, matching the most vulnerable areas of hypoperfusion in early AD patients [17-19]. Moreover, these mice also display microinfarctions, microbleeds, cerebral amyloid angiography, and hippocampus-dependent neurodegeneration in an age-dependent manner. However, another invariant feature of VCID patients, namely, white matter pathology, was not studied previously.

White matter health is of paramount importance to normal cognitive performance [20]; the recent comprehensive transcriptomic study on AD forebrain using single-cell RNA sequencing on different cell types revealed that genes associated with the myelination pathways are the most changed group correlated with early stages of disease progression [21]. White matter tracts are a region at heightened risk for vascular damage [22]. The exact molecular and cellular mechanisms of white matter changes are unclear. Chronic hypoperfusion, blood-brain barrier (BBB) breakdown, oxidative stress, and inflammation have all been postulated as potential mechanisms [23-26]. In this study, we present data on all these aspects of cellular events concerning white matter changes in the eNOS-deficient mouse model.

\section{Methods}

\section{Materials And Methods}

\section{Animals}

Mice deficient in eNOS gene expression (eNOS ${ }^{-/-}$, NOS3 ${ }^{\text {tm1 } 1 \text { Unc }} / \mathrm{J}$ Jackson Laboratory, Bar Harbor ME) were crossbred with C57BL/ 6 mice to obtain eNOS ${ }^{+/-}$. Animals eNOS ${ }^{+/-}$, eNOS ${ }^{-/-}$and littermate eNOS ${ }^{+/+}$ mice were derived from interbreeding between $\mathrm{eNOS}^{+/-}$mice. Littermate mice were genotyped by genomic PCR using tail biopsy samples. For sodium nitrate (SN) feeding, $\mathrm{NaNO}_{3}$ (S5506, Sigma, St. Louis, MO, USA) was added to the drinking water at a concentration of $85 \mathrm{mg} \cdot \mathrm{L}^{-1}(1 \mathrm{mM})$ [27]. All mice were housed and aged in well-ventilated cages under standard laboratory conditions on a 12:12 hour light-dark cycle with food and water ad libitum. All experimental animal procedures were conducted in 
accordance with the animal care standards of the National Institute of Health and were approved by the Institutional Animal Care and Use Committee of the University of Tennessee Health Science Center.

\section{Immunohistochemistry}

Mice were perfused transcardially with ice-cold phosphate buffered saline (PBS) followed by ice-cold $4 \%$ paraformaldehyde (PFA) solution in PBS. Mouse brains were then removed from the cranium. After postfixation in $4 \%$ PFA in PBS at $4^{\circ} \mathrm{C}$ overnight, brains were either processed for OCT-embedding after passing through $20 \%$ to a final of $30 \%$ sucrose solution prepared in PBS for $36-48 \mathrm{~h}$ at $4^{\circ} \mathrm{C}$ until fully sank or transferred to PBS for vibratome sectioning of "fresh" tissue. Brains were then cut into serial coronal sections by Cryostat (CM1900; Leica, Heidelberger, Germany) $(16 \mu \mathrm{m})$ or $30 \mu \mathrm{m}$ thickness on a vibratome for immunostaining after permeabilization with $0.1 \%$ Triton X-100 (v/v) in $0.01 \mathrm{M} \mathrm{PBS}(\mathrm{pH} 7.4)$ for 15 minutes, followed by blocking in 10\% normal goat serum (NS02L; Sigma-Aldrich, Inc.) for 1 hour at room temperature. Table 1 summarizes the primary antibodies used in immunohistochemistry. After incubation with primary antibodies diluted in blocking solution overnight at $4^{\circ} \mathrm{C}$, tissue sections were washed in PBS and incubated with the following secondary antibodies diluted in blocking solution for 60 min at room temperature: Alexa Fluor ${ }^{\circledR} 594$ or 488 conjugated goat anti-rabbit or mouse $\lg (\mathrm{H}+\mathrm{L})($ Cat. A11037, A11032, A11034, A11029; 1:1,000; Thermo Fisher Scientific, Inc.). Sections were washed in PBS and counterstained with DAPI (Roche Diagnostics, Lot\# 70237122, 1:1000). After washing with PBS three times, sections were mounted on microscopy slides and covered with Fluoromount-G ${ }^{\mathrm{TM}}$ (Cat. 00-4959-52; Thermo Fisher Scientific, Inc.). Fluorescence images were captured by inverted fluorescence microscopy (Olympus IX50, Olympus Optical, Inc.) or Keyence's All-in-One fluorescence microscope (BZ-X800E). Fluorescent signals were quantified by NIH's Image $\mathrm{J}$ software.

\section{Histochemistry}

Luxol Fast Blue (LFB) staining was performed following the manufacture's protocol (NovaUltra Luxol Fast Blue Stain Kit, Cat IW-3005, IHC World). To measure brain reactive oxygen species (ROS) production, the brain sections were exposed to dihydroethidium (DHE) (D1168; Thermo Fisher Scientific, Inc.), according to a previously published validated method. Briefly, brain sections were incubated in PBS for 30 minutes at $37^{\circ} \mathrm{C}$ and then encircled with the hydrophobic pen. DHE ( $\left.5 \mathrm{mmol} / \mathrm{L}\right)$ was topically applied, followed by incubation at $37^{\circ} \mathrm{C}$ in the dark for 30 minutes. Sections were rinsed in PBS, and oxidized DHE fluorescence was detected with an Olympus inverted fluorescence microscopy, and the fluorescence staining was quantified by ImageJ.

\section{Cerebral fluorescent angiography}

Cerebral fluorescein isothiocyanate (FITC)-dextran fluorescent angiography was performed as described in detail previously [16] using FITC-dextran (FD2000S-1G; 2000 kDa; $0.1 \mathrm{~mL} 50 \mathrm{mg} / \mathrm{mL}$ in sterile saline; Sigma, St. Louis, MO, USA). Mice were euthanized without perfusion 5 min after injecting FITC-dextran via tail vein and whole brains removed and post-fixed in $4 \%$ PFA in PBS at $4^{\circ} \mathrm{C}$ overnight. Sequential coronal sections (100 mm thickness) were processed using vibratome for fluorescent imaging.

\section{Evans blue angiography}


Evans Blue (E2129, Sigma, St. Louis, MO, USA) was injected in $150 \mathrm{ml}$ of $2 \%(\mathrm{~m} / \mathrm{v})$ in sterile saline through mouse tail vein. Mice were euthanized 5 min later without perfusion and whole brains removed and post-fixed in $4 \%$ PFA in PBS at $4^{\circ} \mathrm{C}$ overnight. Sequential coronal sections (100 mm thickness) were processed using vibratome for fluorescent imaging using rhodamine filter of inverted fluorescence microscopy (Olympus IX50, Olympus Optical, Inc.). Serial images were taken at Bregma: $2.0 \mathrm{~mm}, 1.42$ $\mathrm{mm}, 0.26 \mathrm{~mm},-0.22 \mathrm{~mm}$, and $-1.82 \mathrm{~mm}$. For cerebral double fluorescent angiography, mice received a mixture of FITC-dextran and Evans blue dye in $200 \mathrm{ml}$ volume and euthanized $5 \mathrm{~min}$ later without perfusion. Brain tissue was processed for individual angiography.

\section{White matter micro punch}

Mice were euthanized by isoflurane overdose and brains were excised and placed in ice-cold PBS. Brains were subsequently sectioned with a vibratome to obtain two $1 \mathrm{~mm}$ coronal sections containing the genu and body of the corpus callosum. White matter microsamples were collected via micro punch using a 0.5 $\mathrm{mm}$ blunt-end needle attached to an air-filled syringe under a dissecting microscope. Micro punches of the corpus callosum were collected and aspirated into microcentrifuge tubes in $0.5 \mathrm{ml}$ TRIzol (Invitrogen) and stored at $-80^{\circ} \mathrm{C}$ until RNA extraction. The corresponding size of gray matter tissue was collected via micro punch from the adjacent subcortical region as control.

\section{Western blot analysis and quantitative real-time RT-PCR (qRT-PCR)}

Western blots and qPCR were performed as described previously [16]. Briefly, mice were transcardially perfused with ice-cold PBS and brains were removed. Half brains were post-fixed and used in immunohistochemistry and the other half brains were further dissected to forebrain tissue and then homogenized in cell lysis buffer (20 mM Tris-HCl (pH 7.5), $150 \mathrm{mM} \mathrm{NaCl}, 1 \mathrm{mM} \mathrm{Na} 2 \mathrm{EDTA}, 1 \%$ NP-40, 1\% sodium deoxycholate $0.1 \%$ SDS) with complete protease inhibitor cocktail (Roche, Inc.). Lysate preparation and Western blot analysis were performed as previously described [16]. For qPCR, RNA was extracted from the dissected brain tissue using TRIzol according to the manufacturer's instructions. Single-stranded cDNA was synthesized from $1 \mathrm{mg}$ of total RNA using High Capacity cDNA Reverse Transcription kits (Applied Biosystems). qPCR was performed with RealMasterMix SYBR Green (Applied Biosystems). Primary antibodies and qPCR primers used are summarized in Tables 2 and 3, respectively.

\section{Gait balance test (CatWalk)}

Mice were subjected to gait assessment using the CatWalk (Noldus Information Technology, Wageningen, The Netherlands) which consists of an enclosed walkway on a glass plate that is traversed by mice from one side of the walkway to the other. The room light is off. A camera is set below the glass floor to record paw prints. The red light installed inside the deck generates the background in the video. The glass floor sheds green fluorescence-like light so that paw prints are distinguished when the paw touches the floor. The digitalized paw prints are then automatically classified into four cohorts, namely left forepaw, left hind paw, right forepaw, and left hind paw. Mice from the same housing cage were trained to traverse a glass walkway toward their home cages from one end to another without interruptions for 30 minutes for 
five consecutive days. On subsequent test day, three non-stop complete runs across the walkway were recorded. The test starts once the mouse enters the visual field of the camera and stops once the mouse disappears. If an animal failed to complete a run, walked backwards, or reared during the run, it was given an additional run. Quantitative analysis of gait performance was done by CatWalk XT version 10.0.408 to include the (1) spatial parameters related to individual paws (intensity, maximum area, print area, etc); (2) relative spatial relationship between different paws (base of support, relative paw placement, and stride length); (3) interlimb coordination (step pattern, regularity index, and phase lag); and (4) temporal parameters (swing, stance, cadence, and walk speed). Data are presented as mean \pm s.e.m.

\section{Statistical analysis}

Statistical analyses were performed using unpaired two-tailed or one-tailed Student's t-test. For Western blot quantification, statistics were performed using one-way ANOVA test. Data are reported as mean \pm s.e.m., except as mean \pm s.d.m. for qPCR. Statistical significance was set at $P<0.05$.

\section{Results}

\section{Middle aged eNOS-deficient mice display severe myelin loss and axonal pathology}

In our previous work, we characterized AD-like pathologies in eNOS-deficient mice at old age [16]. We have recently focused on characterizing middle-aged mice, since metabolic vascular factors pose the highest risk in this age group [5-7]. As reported, eNOS-deficient mice manifested most of the phenotypes of metabolic syndrome such as hypertension, insulin resistance, hyperlipidemia, hypertension, and hyperhomocysteinemia [28-30]. We now determined white matter changes at three ages $(7 \mathrm{MO}, 12 \mathrm{MO}$, and $24 \mathrm{MO})$ in eNOS-deficient ( $\mathrm{eNOS}^{-/-}$) and littermate control mice $\left(\mathrm{eNOS}^{+/+}\right.$. Coronal sections encompassing the brain regions corresponding from frontal to temporal cortical areas [+2.0 $\mathrm{mm}$ to -2.5 $\mathrm{mm}$ ] were systematically examined by histological means for the expressional changes in axonal and myelin protein markers. Firstly, myelin integrity was assessed in eNOS mouse brains by both LFB (luxol fast blue) staining (Fig. 1A) and immunohistochemistry on myelin basic protein (MBP) (Fig. 1B). Significant myelin loss was first detected in the cortex and corpus callosum (CC), but not in the striatum in $\mathrm{eNOS}^{-/-}$mice at 12 months of age (12 MO) (Fig. 1 and SFigure 1). Myelin loss was also detected in eNOS heterozygous mice at mid-age (SFigure 1B) and thinning of the CC region, the largest white matter zone, was detected at old age (24 MO).

Surprisingly, Western blot data based on forebrain tissue lysates showed a nearly 2 -fold increase in MBP protein in eNOS ${ }^{-/-}$at mid-age (12 MO) on all four isoforms from alternative splicing, with $17 \mathrm{kDa}$ and $21.5 \mathrm{kDa}$ being the predominant species (SFigure 1C) which presumably play differential roles of functional significance in oligodendrocytes. Notably, there was markedly increased colocalization of MBP immunosignals in the nuclei of eNOS ${ }^{-/-}$brain sections (SFigure 1D). It is unclear if these nucleustranslocated MBP species represent mis-aggregated dysfunctional protein which warrants further investigation via biochemical analysis. Nuclear MBP has been reported to display different roles in myelination compared to cytoplasmic protein species, most often seen with the $17 \mathrm{kDa}$ and $21.5 \mathrm{kDa}$ 
isoforms [31], appearing to be associated with the process during oligodendrocyte maturation before compact myelin formation [32]. Indeed, we observed similar results on two additional myelin protein markers Olig1 (oligodendrocyte transcription factor 1) and CC-1 (mature oligodendrocyte somata marker) (SFigure 2A and 2B), indicating the impaired function of myelin-producing oligodendrocytes which was supported by additional data to be presented later.

\section{Severe gait imbalance and cortical pyramidal layer neurodegeneration were detected in middle aged eNOS-deficient mice}

Cortical white matter change is often associated with gait dysfunction. We used the CatWalk system to assess gait performance of eNOS littermate mice in all three genotypes at 12 months of age. The test started once the mouse entered the visual field of the camera and stopped once the mouse disappeared. The averaged running speed is therefore reciprocal to the duration of the trial. Since the traces of each limb are marked, the moving speed can be alternatively calculated based on each limb. The immediate speed can be constant or vary heavily, so the variability is evaluated as well. We present data in Fig. 2A that the averaged running speed was consistently reduced in $\mathrm{eNOS}^{+/-}$mice and was even slower in $\mathrm{eNOS}^{-/-}$mice. Of note, eNOS ${ }^{-/-}$mice not only moved slowly, but also failed to run at a constant speed compared to $\mathrm{eNOS}^{+/+}$and eNOS ${ }^{+/-}$mice. Moreover, eNOS-deficient mice (both eNOS ${ }^{+/-}$and $\mathrm{eNOS}^{-/-}$) displayed significantly altered patterns of gait sequence inter-limb coordination compared to eNOS ${ }^{+/+}$ mice. The same cohort of eNOS-deficient mice displayed significant deficits in two additional tests of rotarod and grip strength for motor functions at mid-age compared to $\mathrm{NOS}^{+/+}$mice (data not shown).

Cortical white matter demyelination predictably leads to cortical circuit dysfunction. We then examined the overall health of the neurons in both cortical and hippocampal regions of $\mathrm{eNOS}^{-/-}$and control mice by LFB-Nissl double staining. Strikingly we detected disorganized cortical structure with signs of severe neurodegeneration in cortical pyramidal neuronal layers II/III and V/VI at 12 months of age, revealing the presence of an empty vacuole surrounding the seemingly degenerated neurons due to loss of myelin sheath (Fig. 2B). Of note, we did not detect apparent neurodegeneration of hippocampal neurons at this age. Consistently, we detected a significant increase in the expression of activated caspase-3 in the forebrain samples of eNOS ${ }^{-/-}$mice compared to control littermate eNOS ${ }^{+/+}$forebrain (SFigure 2C). Notably, myelin loss appeared to occur before the cortical pyramidal neurodegeneration since no neurodegeneration was detected at 7 months of age while there was a sign of demyelination (SFigure 3).

\section{Elevated ROS and astrogliosis and neuroinflammation detected selectively in the white matter before the middle age}

Since myelin-producing oligodendrocytes are the most energy-demanding cell type in CNS at the highest metabolic rate, they are known to be most vulnerable to stress such as ROS and hypoxia/ischemia. Indeed, we observed markedly elevated ROS levels by DHE staining, selectively in the parietal cortical region (Fig. 3A); no significant increase in DHE staining signal in the CC or striatal brain regions at middle age (SFigure $4 \mathrm{~A})$. 
Furthermore, we found markedly increased fibrous astrocytes based on the expression of the glial fibrillary acidic protein (GFAP) in the CC region in middle-aged eNOS-deficient mice; they were aligned in rows between the axon bundles in parallel which displayed smooth and long processes of a fingerlike shape (Fig. 3B). Heterozygous eNOS ${ }^{+/-}$brains also displayed an increase in GFAP positive cells at 12 months of age, but at lesser degrees than $\mathrm{eNOS}^{-/-}$brains (SFigure 4B). Surprisingly, there was no significant increase in Iba-1 positive microglia in the brains of 12 months-old eNOS-deficient mice; a significant global increase was found at 24 months of age (Fig. 4C). It remains to be determined the precise order of these events at molecular and cellular levels.

Although GFAP is the main astrocytic intermediate filament, immunohistochemistry using multiple GFAP antibodies only labeled astrocytes in the corpus callosum, cerebral peduncle, and the hippocampus in mouse brain under physiological condition. Owning to the increasingly recognized heterogeneity of astrocytes from different brain regions [33], we used a second marker S100b which is more suitable for capturing the overall distribution of astrocytes in all brain regions. Indeed, we observed more S100bpositive astrocytes in the cortical area in $\mathrm{eNOS}^{-/-}$brain at mid-age; not only the number but also the fluorescent intensity of each astrocyte was markedly increased (SFigure 4C). Most strikingly, contrary to the globally increases S100b-positive astrocytes in both the $\mathrm{CC}$ and cortical areas in $\mathrm{eNOS}^{-/-}$brains, the GFAP-positive astrocytes were selectively found around the cortical layer II/III and V/VI regions, surrounding the degenerating/degenerated pyramidal neurons (SFigure 4D). Astrocytes are best known for multi-faces, in tissue repair, and in mediating an inflammatory response. Indeed, at higher magnification, both the GFAP- and S100b-positive astrocytes displayed thicker cellular process, indicating reactive astrocytes upon injury (SFigure4C and 4D).

To verify that these newly increased astrocytes are indeed reactive astrocytes upon injury, we undertook a different approach of investigating cytokine gene expressional changes from the largest CC region (white matter, WM) and the adjacent subcortical region (gray matter, GM) (Fig. 4A). Most strikingly, markedly upregulated levels of three pro-inflammatory cytokine genes were detected only in the white matter of the $\mathrm{eNOS}^{-1-}$ brain at 10 months of age (Fig. 4B). Because we did not observe increased microgliosis at this age, we reasoned that these cytokines were most likely released from the activated reactive GFAP-positive astrocytes. Given the sparse nature of the newly emerged GFAP-astrocytes in the specific regions of cortical areas in $\mathrm{eNOS}^{-/-}$mouse brains by mid-age, the upregulated inflammatory gene expression in gray matter may be oversight from the micro samples taken via micro punch which consisted of a majority of dense neuronal bodies (somas). Nevertheless, the prominently elevated cytokines in the CC region are the unambiguous evidence for neuroinflammation, specifically white matter neuroinflammation, as an early contributing mechanism to the cortical pathology such as demyelination and selective cortical neurodegeneration. Consistently, all four major myelin pathway genes (i.e., Mbp, Plp1, Mog and Mag) were found to be markedly downregulated in the $\mathrm{CC}$ region in eNOS ${ }^{-/-}$mice at 10 months of age as compared to littermate control mice based on quantitative qPCR data (Fig. 4C). The implication of impaired integrity of the myelinated axons and white matter axon-glial coupling upon eNOS deficiency will be discussed later. 


\section{Hypoperfusion and BBB leakage are the two earliest pathologies detected in eNOS-deficient mice}

Endothelium-derived NO (EDNO) signaling has long been known to be crucial for angiogenesis and vasculogenesis during the early developmental stage. Using FITC-angiography, we detected reduced vessels from a dorsal view of eNOS-deficient mice at a young age (3 MO) (Fig. 5A, left two panels), which was similarly reported previously not attributable to impaired collateral formation in the embryo-neonate stage [34]. We therefore reasoned that this phenomena indicated arterial occlusion resulting from impaired NO signaling. Indeed, as we reported previously [16], regions of the hypoperfused brain and microvascular occlusion appear in eNOS-deficient mice at a young age, with markedly increased frequency of similar lesions in an age-dependent manner. As shown in a representative cortical section from young eNOS-deficient mice (3 MO) (the right panel of Fig. 5A), these lesions/occlusions are typically small (ranging from 100 to $200 \mu \mathrm{m}$ in diameter); by middle age, they are frequently found bilaterally in the parietal association cortex (Table 1). This pathological pattern is highly reminiscent of the bilateral temporoparietal hypoperfusion characteristic of AD patients. Occlusions were not uniformly located throughout the $\mathrm{eNOS}^{-/-}$brain, but rather in defined areas, listed in order of higher to lower frequency: parietal association, temporal association and retrosplenial granular cortices, hippocampus, and thalamus. No such lesions were detected in the WT eNOS littermates up to 24 months of age.

In our prior work we also reported compromised BBB in middle to old-aged eNOS-deficient mice based on FITC-dextran (150 kDa) angiography as well as quantitative mouse Ig extravasation [16]. We speculated that BBB integrity and function may be compromised at a much younger age in eNOS-deficient mice owning to the indispensable role of EDNO signaling in endothelial vascular permeability via modulating VEGF signaling. To detect early BBB leakage, we systematically tested various molecular tracers reportedly used for this purpose including FITC-dextran species at different molecular weights and Evans blue dye. Surprisingly, when we used FITC-dextran of a lower molecular weight range (3-30 kDa) as a tracer, fluorescence was not detected in the brain, presumably due to rapid systemic clearance. In the end, we concluded that Evans blue dye is an optimal fluorescent tracer for the degrees of leakage in the eNOS model.

Evans blue (980 Da) is an azo dye with a very high affinity for serum albumin $(67 \mathrm{kDa})$. Because serum albumin cannot cross the BBB and virtually all Evans blue is bound to albumin, albumin-bound Evans blue enters the parenchymal tissue in CNS when the BBB has been compromised. Since Evans blue fluoresces with excitation peaks at 470 and $540 \mathrm{~nm}$ and an emission peak at $680 \mathrm{~nm}$, it can be detected in brain sections under a fluorescent microscope using a Rhodamine filter. After injecting Evans blue dye to mouse tail vein, the brains were removed shortly and post-fixed and processed to serial $100 \mathrm{~mm}$ coronal sections for angiographic examination. By this method, we detected massive BBB leakage in eNOS $^{-/-}$mice beginning at a very young age (6-8 week-old). As shown in Fig. 5B, eNOS-deficient brain at 3 months of age displayed numerous diffusive spots indicating BBB leakages spanning from frontal to parietal cortical regions compared to no leakage found in littermate control mice. The gradually decreased leakage spots in the five representative angiographs spanning Bregma $+2.0 \mathrm{~mm}$ to $-1.82 \mathrm{~mm}$ indicates that leakages start predominantly from an anterior to posterior direction. Indeed, we detected 
more severe leakage expanded to deeper and posterior areas in older eNOS-deficient mice. Strikingly, like FITC-non-perfusion lesions, there was no leakage detected in wild-type eNOS mice even at 24 months of age using this methodology (Table 1). Interestingly, we examined mouse brain sections after co-injection of FITC-dextran (2000 kDa) and Evans blue dye and found largely non- overlapping fluorescent signals in $\mathrm{eNOS}^{-/-}$brain at a young age (data not shown), suggesting that these two early pathological events of non-perfusion and BBB leakage are independent of each other, both resulting from the impaired EDNO signaling.

Accordingly, we detected massive upregulation of metalloproteinases 9 (MMP9) at middle age by both immunohistochemistry and Western blot analysis, with MMP9-positive signals largely colocalizing with the Evans blue leakage signals (SFigure 5A-C), indicating breakdown of the endothelial basement membrane. Surprisingly, we detected no significant changes in MMP2 expression, and laminin expression was only elevated at 24 months of age (SFigure 5D). Since astrocytes contact blood capillaries with their endfeet as an integral part of the BBB interface, they are crucial in governing and maintaining water homeostasis in the CNS. We therefore also investigated aquaporin-4/Aqua4, a marker of astrocytic endfeet surrounding the capillaries by immunohistochemistry. Aqua4 proteins displayed more intense expression in microvessels in gray matter than in white matter, consistently because the microvessel density is much lower in white matter. Although Western blot data showed no significant change in Aqua4 protein expression levels from forebrain tissues of eNOS ${ }^{-/-}$mice at 12 months of age, Aqua4 proteins appeared to undergo redistribution upon eNOS deficiency by middle age; there were slightly more intense Aqua4 immunosignals overlapping with GFAP-positive astrocyte endfeet along the microvessels (SFigure 6B), suggesting altered BBB functions. Western blot analysis revealed nearly complete loss of Aqua4 protein expression in old eNOS ${ }^{-/-}$mouse brain at 24 months of age (SFigure 6C). Similarly, we detected a significant reduction of glucose transporter 1/GLUT1 expression only at an older age (SFigure $6 \mathrm{D})$.

\section{Restoration of NO signaling via sodium nitrate (SN) feeding rescues white matter pathologies in eNOS- deficient mice}

Restoration of NO signaling with $1 \mathrm{mM} \mathrm{SN}$ in drinking water for up to 10 weeks has been reported to reverse hypertension, systemic hyperlipidemia, and glucose intolerance in 14- to 22-month-old eNOS ${ }^{-/-}$ mice [27]. This prompted us to use this agent to test our hypothesis that feeding eNOS-deficient mice with $\mathrm{SN}$ in drinking water at a very young age, starting from 6-week-old for 6 weeks, would prevent the development of the two early pathological events namely hypoperfusion and BBB leakage. Strikingly, 6week SN feeding completely prevented BBB leakage and non-perfusion lesions in eNOS ${ }^{-/-}$mice (Fig. 5C and Fig. $6 \mathrm{~B}$ and $6 \mathrm{C}$ Cohort 1 mice; $n=3$ /group). These promising data encouraged us to further testing the possibility of chronic feeding sodium nitrate in drinking water to eNOS-deficient mice at older postsymptomatic age.

In addition to the first cohort of mice that received 6-week-SN feeding which resulted in nearly complete prevention of BBB leakage (Fig. $5 \mathrm{C}$ and Fig. 6B), we also fed mice for a longer period in eNOS ${ }^{-/-}$mice for 
over 6 months (Cohort 2) and post-symptomatically (Cohort 3, 11-16 months) (Fig. 6A). We focused on ROS, white matter inflammation, and myelin preservation in the Cohort 2 mice. Specifically, SN feeding completed prevented pro-inflammatory cytokine gene upregulation in eNOS-/- white matter tissue at 10 months of age (Fig. 4B) and the decline of myelin pathway gene expression (Fig. 4C). It also prevented ROS elevation and astrogliosis in selective cortical layers (data not shown). Furthermore, loss of myelin neurofilaments (SMI 132) in eNOS ${ }^{-/-}$brains was significantly rescued by SN feeding (Fig. 6C). Lastly, gait performance was significantly improved in Cohort 3 mice when tested at 16 months of old age, after SN feeding was initiated from 11 months of age in $\mathrm{eNOS}^{-/-}$mice (Fig. 6D). Although the SN eNOS ${ }^{-/-}$mice could not perform as well as the eNOS ${ }^{+/+}$mice at this old age (16 MO), this result is most encouraging with translational value for a potential reversal effect of cortical neuronal connectivity and functions. Taken together, chronic SN feeding resulted in significant prevention or improvement in white matter pathologies and function in $\mathrm{eNOS}^{-/-}$mice.

\section{Discussion}

White matter comprises half of the brain and has expanded more than gray matter during evolution, continuing from childhood through adulthood. Over the past decade, white matter is increasingly recognized as equally critical as the cerebral cortex and hippocampus for cognition. This is not surprising given the fact that white matter tracts form an indispensable component of the complex neural connectivity that supports neurobehavioral operations. Nowadays, there is compelling evidence supporting that white matter lesions disturb and probably directly contribute to cognitive dysfunctions [35] .

Myelination, a process that continues for decades in the human brain is crucial for information processing by regulating the velocity and synchrony of axonal impulse conduction between distant cortical regions. It is now well recognized that the process of myelination can be modifiable by experience $[36,37]$. Compelling evidence collected from aging and AD brains suggests that demyelination within the cerebral white matter is an upstream event that initiates amyloid deposition and then tau as later end products comprising cortical pathology in AD [38]. Although this "myelin model" was initially instated in the context of $A D$, the same theory may be generalized to other neurodegenerative and psychiatric conditions including vascular dementia. Thus far, this model has been largely supported by compelling evidence including recent genetic data [21], which is in line with the term "white matter dementia" brought up two decades ago to highlight the importance of white matter dysfunction to cognitive decline. The tight association between the degrees of dementia with the in involvement of white matter lesions [39] provides additional strong support to this theory. Since dementia has been most extensively studied in the form of Alzheimer's type, our current therapeutic development towards dementia is still dominated by anti-Ab/tau strategies with all trials without success in clinics. If the myelin model proves to be correct as a unifying theory for all types of dementia including cortical, white matter and subcortical dementia, it would be instrumental in redirecting our focus from amyloid and tau to myelin-oriented preventive and treatment intervention. Therefore, a deeper understanding of the mechanisms of 
demyelination/remyelination at both cellular and molecular levels would be fundamentally important in designing novel targeting strategies long before the onset of cognitive dysfunction. This relies not only on the knowledge gained from clinical neurology (e. g., neuroimaging and neuropathology) to identify early white matter pathology but also from various representative experimental models of dementia conditions to validate the earliest white matter mechanisms that drive cognitive decline.

This report presents evidence that genetic eNOS-ablation spontaneously induces chronic hypoperfusion, BBB leakage, elevated ROS/astrogliosis, myelin loss, and axonal pathology as well as cortical neurodegeneration/gait imbalance in mice. All these phenotypes are considered the essential components as potential contributing mechanisms to dementia, based on both clinical [20,22-26] and experimental $[40,41]$ evidence. Clinically, dementia has been most widely studied in AD type so-called cortical dementia, the other two types of dementia (i.e., subcortical dementia, often seen with stroke patients), and white matter dementia are featured by distinct core cognitive dysfunctions, especially at the early stages of dementia, likely due to different white matte lesions [35]; of note, these features become indifferent as neuropathologies progress to late stages, all three types of dementias become indistinguishable in the end. Again, these clinical notions underscore the paramount importance of studying white matter myelin mechanisms using representative experimental models.

In addition to the transgenic/knock-in rodent models based on mutant familial AD genes (e.g., APP, PS1, and tau) in which white matter dysfunction was occasionally reported, there are currently more than a dozen of rodent models that develop major features of human dementia with the vascular origin [42]; none of a single model recapitulates all features of human dementia. The optimal choice of model depends on the aspect of pathophysiology being studied. Chronic cerebral hypoperfusion has been suggested to be a key mechanism leading to dementia [15]. Over the last decade, bilateral common carotid occlusion/stenosis (BCAS) has been the most widely used chronic hypoperfusion model. This BCAS surgery in both rats and mice induces several major white matter changes including reduced parenchymal cerebral blood flow, oligodendrocyte loss, glial activation, and disrupted clearance of intracerebral fluid and BBB, and cognitive impairment [43]. However, sizable intrinsic variance in cerebral hypoperfusion with blood flow dropping too suddenly and too severely after occlusion surgery is one major shortcoming of this type of surgical model. Moreover, all pathological events occur within 2-3 months, making the model difficult to consider the aging component. In our opinion, the spontaneously developed white matter and cognitive phenotypes in an age-dependent manner in the mouse model based on eNOS-deficiency (both heterozygous eNOS and eNOS knock-out mice) makes these mice ideal for studying white matter mechanisms, linking chronic hypoperfusion and demyelination directly to neurodegeneration. Below, we will discuss in detail our findings based on the eNOS-deficient mice.

\section{White matter changes, including myelin loss and axonal pathology}

The largest white matter tract, the corpus callosum (CC), is particularly vulnerable to stress. This region has been extensively examined and reported for various pathological changes in the brains of healthy 
elderly individuals, $A D$, multiple sclerosis and vascular dementia. Although we observe $C C$ atrophy in $\mathrm{eNOS}^{-/-}$brain at very old age (24 MO), we detect demyelination in cortical and CC regions, but not striatum, at a much younger age (starting from 7 months of age). The mechanism for CC atrophy is poorly understood, resulting from axonal disruption due to white matter damage.

Maintenance of the integrity of the myelinated axons and the functional coupling between axon and the responsible glial cells (i.e., oligodendrocytes) are well-known to be critical for action potential propagation and thus for regulating efficient neuronal connectivity. Axonal dysfunction in white matter disorders is well recognized to confer a worse prognosis than that implied by myelin damage alone [40]. White matter axon-glial integrity has been reported to be disrupted in mice by mild cerebral hypoperfusion in an experimental model (BCSA) [44]. We used the pan-antibody SMI-32 to visualize the distribution of mixed neurofilaments in $\mathrm{eNOS}^{-/-}$brain at middle age and observed significantly reduced immunosignals in multiple regions, including $\mathrm{CC}$ and subcortical areas (Fig. 6C); the thinner and more "chaotic" staining pattern displayed in $\mathrm{eNOS}^{-/-}$brain also indicated reduced axonal diameters and aberrantly sprouting axons derived from cortico-cortical fibers, predicting the loss of synaptic circuitry underlying the basis of memory. Taken together, our data combined between immunohistochemistry and quantitative gene expression by qPCR analysis, we conclude that both the integrity of the myelin sheath (assessed by MBP) and integrity of axons (assessed by SMI-32) are compromised upon eNOS deficiency. Moreover, the reduced expression of the gene encoding for myelin-associated protein MAG (Fig. 4C) also indicates disruption of the axon-glial integrity. We speculate that this axonal pathology occurs early, before the neuronal cell body pathology. Indeed, we observe demyelination at a young age in eNOS ${ }^{-/-}$mice (7 months of age) while no cortical layer pyramidal neurodegeneration is detected at this age. Surprisingly, the demyelination occurs from the outer layers of cortical region to the inner layers (e.g., layer I to $\mathrm{VI}$ ), consistently with the notion that the outer cortical layers II/III are most vulnerable to hypoperfusion in the clinical specimen of both early demented $A D$ and post-stroke brains $[45,46]$.

\section{Chronic hypoperfusion versus BBB dysfunction}

We have collected evidence to identify hypoperfusion and BBB leakage as the two earliest pathological events in $\mathrm{eNOS}^{-/-}$brains from newly weaned mice (6 weeks of age) via FITC and Evans blue angiographies, respectively. We discovered a general pattern through systemic examination of the coronal brain sections from these angiographies for these two events, both expanding from the anterior to the posterior brains in an age-dependent manner. While FITC-dextran non-perfused lesions occur predominantly at the frontal parietal cortical area, Evans blue leakages are present globally in both dorsal and ventral cortical areas. The overall "anterior-to-posterior" hypoperfusion gradient is a reminiscent of the clinical hypoperfused human brains correlating with various stages of dementia $[47,48]$. For example, the dis-executive individuals often display significant hypoperfusion of the left superior, medial frontal and cingulate cortex. In contrast, the amnestic mild cognitive impaired individuals show significant hypoperfusion in the left hippocampus, para-hippocampal gyrus, and fronto-parieto-temporal areas [49]. 
Perhaps limited by the nature of the two angiographic methods we used, we could not determine which of these two events occurs first. Given the different angiographic profiles from FITC-dextran and Evans blue, we posit that they are independent events, both resulting from impaired EDNO signaling. Indeed, we observe non-overlapping fluorescent signals from the brains receiving a co-injection of both dyes. However, the main question remains of how these initial pathological events lead to the subsequent white matter pathologies. White matter is long speculated to be most vulnerable to chronic hypoperfusion due to intrinsic lower microvessel density $[11,26]$. Despite a recent report excluding BBB leakage as an invariant feature of the white matter lesions based on case studies from the genetic paradigm of small vessel disease so called cerebral autosomal dominant arteriopathy with subcortical infarcts and leukoencephalopathy (CADASIL) [50], more experimental evidence supports a prevailing view that disrupted BBB can contribute directly to white matter changes. For example, using a model of pericytedeficiency, white matter pathology and impaired executive functions were detected. In this model study, oligodendrocyte degeneration was suggested to be caused by an accumulation of toxic blood-derived fibrin(ogen) deposits and blood-flow reductions; the latter further leads to a loss of oligodendrocytes, and thus myelin, and axonal structure and function [39]. Our data are consistent with the observations from this study: both the accumulation of fibrin(ogen) deposits [16,51], as well as a reduced expression of immature and mature oligodendrocyte markers (Oligo1 and CC1) (SFigure 2), are detected in the eNOS model. In addition, oligodendrocyte health, as the most vulnerable cell type in CNS, may also be affected by elevated oxidative stress and inflammatory environment in white matter, in particular, as discussed in the next section.

\section{ROS, astrogliosis and white matter neuroinflammation-what causes demyelination?}

Astrocytes contact blood capillaries with their end feet and are therefore an important part of the bloodbrain interface, presenting differences between gray (GM) and white matter (WM). Interestingly, astrocytes exert different properties of protoplasmic and fibrous subtypes in GM and WM, respectively. Therefore, it is likely that these different astrocyte subtypes contribute to the observed differences in BBB properties in GM and WM. We detect marked BBB leakage in eNOS ${ }^{-/-}$brain at a very young age, followed by increased expression of GFAP-positive reactive astrocytes in the CC region and selected cortical layers II/III and V/VI by mid-age. Given the exclusive upregulated pro-inflammatory cytokine gene expression in the $\mathrm{CC}$ white matter region, we reason that the increased astrogliosis in the $\mathrm{CC}$ region likely contribute directly to oligodendrocyte degeneration and demyelination while the sparse presence of the GFAPastrocytes in the cortical areas surrounding the degenerated layer II/III and V/VI pyramidal neurons likely reflect the well-recognized role of astrocyte in tissue repair upon injury. The precise astroglial mechanisms in various pathogenic stages of the white matter changes in the eNOS model will be further investigated at cellular and molecular levels. Our finding of the upregulated genes of the transforming growth factor-beta (TGFb) super-family (i.e., TGFb-1 and BMP4; Fig. 4D) selectively in white matter tissue opens up a new window of investigating impaired signaling of the TGFb family as a potentially crucial glial-vascular mechanism of the white matter changes in the eNOS model. Indeed, we observe 
upregulation of BMP4 expression in the descending arterioles in eNOS ${ }^{-/-}$brain at a young age [51], likely from pericytes as reported from mice undergoing BCAS [52]. Accordingly, the well-known paracrine actions of BMP4 signaling could directly trigger white matter degeneration via promoting astrogliosis and simultaneously interfering with oligodendrocyte differentiation, and maturation; a theory is being tested experimentally. How an impaired EDNO signaling induces upregulated TGFb-1 and BMP4 signaling is also an interesting and challenging question to be addressed.

\section{NO-based therapy - inorganic nitrate/nitrite}

In humans, EDNO plays a key role in regulating basal cerebral blood flow via vasodilation of cerebral vessels, and aberrant biogenesis of NO is associated with arterial stiffness, hypertension, atherosclerosis in patients with cardiovascular diseases, $A D$ and vascular dementia patients [51]. Our data demonstrate that impaired EDNO signaling can first lead to cerebral hypoperfusion and the impairment of the BBB, subsequently to white matter changes and neurodegeneration from cortical to hippocampal regions. Developing NO-based therapeutics thus represents a viable strategy for treating white matter dementia.

Since the seminal discoveries of $\mathrm{NO}$ as the most crucial endothelial-derived relaxing factor, and subsequently, the biology of nitrite anion (i.e., the "nitrate-nitrite-NO pathway") [53], developing NO-based therapeutics have been actively pursued. Several mechanisms probably contributed to the decrease in NO bioavailability, including defects in the cascade of NO generation (e.g., eNOS activity) and reduced NO donors. Thus far there are only three agents are approved by FDA for uses in treating acute angina by organic nitroglycerine, $\mathrm{NO}$ inhalation for pulmonary hypertension in neonates and phosphodiesterase inhibitors to prevent the breakdown of the downstream second messenger of NO, cyclic guanosine monophosphate/cGMP [54]. Although the first line cholesterol-lowering drugs statins also demonstrate a potential to enhance the bioavailability of EDNO, principally by up-regulating and activating eNOS $[55,56]$; convincing clinical data is still lacking $[57,58]$. Importantly, like L-arginine supplementation based on the discovery of it being the physiological substrate of eNOS, statins belong to the same category of NOenhancing strategies requiring functional NOS system as a prerequisite. In theory, using strategies independent of a functional NOS system to increase bioavailability of NO production is more ideal.

Historical evidence suggests that using dietary supplements with natural products represents a safest strategy [59]. Numerous experimental evidence indicates that nitrite anion represents an important reservoir of NO bioequivalents to increase tissue NO levels during pathophysiological states in which NO synthesis from the eNOS-mediated enzymatic pathways are unavailable; nitrite anion production and distribution throughout the body can act in an endocrine manner to augment NO bioavailability [60]. Administration of nitrate or nitrite to humans and rodents clearly led to increased NO-like bioactivity from numerous studies. Feeding eNOS-deficient mice with oral sodium nitrite or sodium nitrate for as short as one to several weeks has been demonstrated by independent groups to restore NO signaling and vascular homeostasis $[27,61]$. Our recent data not only reproduces these observations but also expands to the BBB and white matter beneficial effects (Figs. 5 and 6). These promising findings provide additional mechanistic insights to the NO-based therapy, highlighting the paramount importance of the EDNO signaling. 
Although more than a dozen clinical reports revealing significant beneficial effects from administration of nitrate or nitrite to humans in multiple symptoms, including blood pressure, abnormal platelet aggregation, reduced triglycerides/hypercholesterolemia cardiac ischemia, proving absolute safety [54]. It should be noted that so far the majority of the clinical testing in patients used inorganic nitrate. In humans, a normal NO production scheme requires nitrate to first be reduced to nitrite via two electron reduction, followed by another one electron reduction to NO. Inorganic nitrate is nearly inert in humans due to an intrinsic lack of the functional gene to reduce nitrate to nitrite; the conversion relies on symbiotic oral bacteria and host enzymes which is inefficient (5\%) [54]. Therefore, the required conditions for nitrate to have therapeutic efficacy in humans are difficult to meet. On the other hand, nitrite is more efficacious but also slightly more toxic than nitrate. Therefore caution should be taken in terms of using optimal dosing. Both inorganic nitrite and nitrate are naturally abundant in green leafy vegetables and beets which are safe for humans, though it remains to be determined how much daily intake is sufficient to reach therapeutic benefits. Nevertheless, healthy lifestyle to combine dietary supplements and exercise is the key for a safest and most beneficial NO-based therapy.

\section{Conclusions}

White matter lesions can directly contribute to cognitive dysfunctions via dis-coupled glia-vascular functions. Since we observed hypoperfusion and BBB breakdown before myelin loss in white matter, we speculate that the compromised BBB with vascular injury along with oxidative stress and astrogliosis all contribute to the subsequent white matter change. Moreover, we also detect selective neurodegeneration in the superficial cortical layers (II/III and V/VI), likely due to cortical demyelination. The chronically evolving nature of the spontaneous model of hypoperfusion in eNOS-deficient mice will allow us to tease out the sequential pathological events in a future longitudinal study, at both molecular and cellular levels. These mice offer a unique opportunity to generalize and further validate the "myelin model" in a mouse system without being compounded by the amyloid and tau as dominating factors. We conclude that eNOS-deficient mice may represent an ideal spontaneous model for studying the earliest events leading to white matter changes. Moreover, it would be useful for future testing drug candidates for therapeutic use via targeting novel/specific vascular mechanisms contributing to VCID and AD.

\section{Abbreviations}

Vascular cognitive impairment and dementia (VCID), Alzheimer's disease (AD), Endothelial nitric oxide synthase (eNOS), Endothelium-derived nitric oxide (EDNO), Blood-brain-barrier (BBB), Corpus callosum (CC), White matter (WM), Gray matter (GM), Bilateral common carotid artery stenosis (BCAS).

\section{Declarations}

Ethical Approval and Consent to participate: All animal work had been reviewed and approved by the Institutional Animal Care and Use Committee of the University of Tennessee Health Science Center, and was conducted in accordance with the animal care standards of the National Institute of Health. 
Consent for publication: All authors read and approved the final manuscript.

Availability of supporting data:We will make all materials and data including supporting data available to scientific community upon acceptance of this manuscript.

Competing interests:The authors declare that they have no competing interests.

Funding:This work was supported in part by Alzheimer Foundation ZEN-16-362441 to F. L. L., NIH grants R21 AG041934, NS091593, R01 AG058467and R01 NS120327 to F. L. L., NIH grants R01 NS097800 to T. I., Joint Funds for the Innovation of Science and Technology of Fujian Province, China (2017Y9065) and High-level Hospital Foster Grants from Fujian Provincial Hospital, Fujian province, China (2020HSJJ07) to X-Y C.

Authors' contributions: Drs. X. Y. C., L. C., G. L., W. Z., M. C. K. and S. G. L. performed the experiments and analyzed data; L. X. L. and S. I., T.C. O. and P. S. assisted in data analysis. F. F. L. and K. U. M. directed the project and F. F. L. wrote the manuscript.

Acknowledgements: We specially thank Dr. Lubin Lan, Ms Kadijah Wainwrigh and Ms Sherry E Frazier Warford for providing excellent technical support; Drs. David Hamilton, Brianne M. Hibl and Samuel Tyler Aycock for general consultation on animal welfare. We also thank Dr Xing-Lin Tan (Nanhai Hospital of Southern Medical University, China) and Dr. Jon O Lundberg (Karolinska Institute, Sweden) for constructive discussion.

Authors' information: All authors current addresses are listed on the cover page under affiliation.

\section{References}

1. Snyder HM, Corriveau RA, Craft S, Faber JE, Greenberg S, Knopman D, et al. Vascular Contributions to Cognitive Impairment and Dementia Including Alzheimer's Disease. Alzheimer's Dementia. 2015;11:710-7.

2. Schneider JA, Arvanitakis Z, Bang W, Bennett DA. Mixed brain pathologies account for most dementia cases in community-dwelling older persons. Neurology. 2007;69:2197-204.

3. Iadecola C. The pathobiology of vascular dementia. Neuron. 2013;80:10.

4. Attems J, Jellinger K. The overlap between vascular disease and Alzheimer's disease lessons from pathology. BMC Med. 2014;12:206.

5. Hofman A. Ott A, Breteler MM, Bots ML, Slooter AJ, van Harskamp F, et al. Atherosclerosis, apolipoprotein $\mathrm{E}$, and prevalence of dementia and Alzheimer's disease in the Rotterdam study. Lancet. 1997;349:151-4.

6. Bhat NR. Linking cardiometabolic disorders to sporadic Alzheimer's disease: a perspective on potential mechanisms and mediators. J Neurochemistry. 2010;115:551-62. 
7. Dickstein DL, Walsh J, Brautigam H, Stockton SD Jr, Gandy S, Hof PR. Role of vascular risk factors and vascular dysfunction in Alzheimer's disease. Mt Sinai J Medicine. 2020;77:82-102.

8. Johnson NS, Jahng GH, Weiner MW, Miller B, Chui HC, Jagust WJ, et al. Pattern of cerebral hypopersufion in Alzheimer disease and mild cognitive impairment measured with arterial spinlabeling MR imaging: initial experience. Radiology. 2005;234:851-59.

9. de la Torre JC. Cardiovascular Risk Factors Promote Brain Hypoperfusion Leading to Cognitive Decline and Dementia. Cardiovascular Psychiatry Neurology. 2012;2012:367516.

10. Iadecola $\mathrm{C}$. The overlap between neurodegenerative and vascular factors in the pathogenesis of dementia. Acta Neuropathology. 2010;120:287-96.

11. Brown WR, Thore CR. Review: cerebral microvascular pathology in ageing and neurodegeneration. Neuropathol Appl Neurobiology. 2011;37:56-74.

12. Minoshima S, Giordani B, Berent S, Frey KA, Foster NL, Kuhl DE. Metabolic reduction in the posterior cingulate cortex in very early Alzheimer's disease. Ann Neurology. 1997;42:85-94.

13. Mosconi L, Pupi A, De Leon MJ. Brain Glucose Hypometabolism and Oxidative Stress in Preclinical Alzheimer's Disease. Ann N Y Acad Science. 2008;1147:180-95.

14. Mustapha M, Nassir CMNCM, Aminuddin N. Safri AA, Ghazali MM. Cerebral Small Vessel Disease (CSVD) - Lessons From the Animal Models. Front Physiology. 2019;10:1317.

15. Duncombe J, Kitamura A, Hase Y, Ihara M, Kalaria RN, Horsburgh K. Chronic cerebral hypoperfusion: a key mechanism leading to vascular cognitive impairment and dementia. Closing the translational gap between rodent models and human vascular cognitive impairment and dementia. Clin Science (Lond). 2017;131:2451-68.

16. Tan XL, Xue YQ, Ma T, Wang X, Li JJ, Lan L, et al. Partial eNOS deficiency causes spontaneous thrombotic cerebral infarction, amyloid angiopathy and cognitive impairment. Mol Neurodegeneration. 2015;10:24.

17. Luckhaus C, Flüb MO, Wittsack HJ, Grass-Kapanke B, Jänner M, Khalili-Amiri R, et al. Detection of changed regional cerebral blood flow in mild cognitive impairment and early Alzheimer's dementia by perfusion-weighted magnetic resonance imaging. Neuroimage. 2008;40:495-503.

18. Schuff N, Matsumoto S, Kmiecik J, Studholme C, Du A, Ezekiel F, et al. Cerebral blood flow in ischemic vascular dementia and Alzheimer's disease, measured by arterial spin-labeling magnetic resonance imaging. Alzheimers Dementia. 2009;5:454-6.

19. Hu WT, Wang Z, Lee VM, Trojanowski JQ, Detre JA, Grossman M. Distinct cerebral perfusion patterns in FTLD and AD. Neurology. 2010;75:881.

20. Longstreth WTJr, Manolio TA, Arnold A, Burke GL, Bryan N, Jungreis CA, et al. Clinical correlates of white matter findings on cranial magnetic resonance imaging of 3301 elderly people. The Cardiovascular Health Study Stroke. 1996;27:1274-82.

21. Mathys H, Davila-Velderrain J, Peng Z, Gao F, Mohammadi S, Young JZ, et al. (2019) Single-cell transcriptomic analysis of Alzheimer's disease. Nature. 2019;570:332-7. 
22. Black S, Gao FQ, Bilbao J. Understanding White Matter Disease Imaging-Pathological Correlations in Vascular Cognitive Impairment. Stroke. 2009;40[suppl 1]:S48-S52.

23. The LADIS Study Group. Poggesi A, Pantoni L, Inzitari D, Fazekas F, Ferro J, O'Brien J, et al. 20012011: a decade of the LADIS (Leukoaraiosis And DISability) study: what have we learned about white matter changes and small-vessel disease? Cerebrovasc Disease. 2011;32:577-88.

24. Rosenberg GA. Inflammation and White Matter Damage in Vascular Cognitive Impairment. Stroke. 2009;40:20-3.

25. Fernando MS, Simpson JE, Matthews F, Brayne C, Lewis CE, Barber R, et al. White matter lesions in an unselected cohort of the elderly: molecular pathology suggests origin from chronic hypoperfusion injury. Stroke. 2006;37:1391-8.

26. Hase $Y$, Horsburgh $K$, Ihara $M$, Kalaria RN. White matter degeneration in vascular and other ageingrelated dementias. J Neurochemistry. 2018;144:617-33.

27. Carlström M, Larsen FJ, Nystrom T, Hezel M, Borniquel S, Weitzberg E, et al. Dietary inorganic nitrate reverses features of metabolic syndrome in endothelial nitric oxide synthase-deficient mice. Proc Natl Acad Science U S A. 2010;107:17716-20.

28. Duplain H, Burcelin R, Sartori C, Cook S, Egli M, Lepori M, et al. Insulin Resistance, Hyperlipidemia, and Hypertension in Mice Lacking Endothelial Nitric Oxide Synthase. Circulation. 2001;104:342-5.

29. Cook S, Hugli O, Egli M, Vollenweider P, Burcelin R, Nicod P, et al. Clustering of cardiovascular risk factors mimicking the human metabolic syndrome X in eNOS null mice. Swiss Med Wkly. 2003;133:360-3.

30. Zintzaras E, Kitsios G, Stefanidis I. Endothelial NO Synthase Gene Polymorphisms and Hypertension: A Meta-Analysis. Hypertension. 2006;48:700 - 10.i.

31. Staugaitis SM, Smith PR, Colman DR. Expression of myelin basic protein isoforms in nonglial cells. J Cell Biology. 1990;110:1719-27.

32. Pedraza L, Fidler L, Staugaitis SM, Colman DR. The active transport of myelin basic protein into the nucleus suggests a regulatory role in myelination. Neuron. 1997;18:579-89.

33. Lundgaard I, Osório MJ, Kress BT, Sanggaard S, Nedergaard M. White matter astrocytes in health and disease. Neuroscience. 2014;276:161173.

34. Dai X, Faber JE. Endothelial nitric oxide synthase deficiency causes collateral vessel rarefaction and impairs activation of a cell cycle gene network during arteriogenesis. Circ Research. 2010;106:187081.

35. Filley CM, Fields RD. White matter and cognition: making the connection. J Neurophysiology. 2016;116:2093-104.

36. Wang F, Ren SY, Chen JF, Liu K, Li RX, Li ZF, et al. Myelin degeneration and diminished myelin renewal contribute to age-related deficits in memory. Nat Neuroscience. 2020;23:481-6.

37. Pan S, Mayoral SR, Choi HS, Chan JR, Kheirbek MA. Preservation of a remote fear memory requires new myelin formation. Nat Neuroscience. 2020;23:487-99. 
38. Bartzokis G, Sultzer D, Lu PH, Nuechterlein KH, Mintz J, Cummings JL. Heterogeneous age-related breakdown of white matter structural integrity: implications for cortical "disconnection" in aging and Alzheimer's disease. Neurobiol Aging. 2004;25:843-51.

39. Filley CM. White matter dementia. Ther Adv Neurol Disorder. 2012;5:267-77.

40. Madigan JB, Wilcock DM, Hainsworth AH. Vascular Contributions to cognitive Impairment and dementia - topical review of animal models. Stroke. 2016;47:1953-9.

41. Montagne A, Nikolakopoulou AM, Zhao Z, Sagare AP, Si G, Lazic D, et al. Pericyte degeneration causes white matter dysfunction in the mouse central nervous system. Nat Medicine. 2018;4:32637.

42. Hainsworth A, Allan SM, Boltze J, Cunningham C, Farris C, Head E, et al. Translational models for vascular cognitive impairment: a review including larger species. BMC Med. 2017;15:16.

43. Washida K, Hattori Y, Ihara M. Animal Models of Chronic Cerebral Hypoperfusion: From Mouse to Primate. Int J Mol Science. 2019;20: 6176.

44. Reimer MM, McQueen J, Searcy L, Scullion G, Zonta B, Desmazieres A, et al. Rapid Disruption of Axon-Glial Integrity in Response to Mild Cerebral Hypoperfusion. J Neuroscience. 2011;31:8185-94.

45. Hof PR, Morrison JH. Quantitative analysis of a vulnerable subset of pyramidal neurons in Alzheimer's disease: II. Primary and secondary visual cortex. J Comp Neurology. 1990;301:55-64.

46. Foster V, Oakley AE,. Slade JY, Hall R, Polvikoski TM, Burke M, et al. Pyramidal neurons of the prefrontal cortex in post-stroke, vascular and other ageing-related dementias. Brain. 2014;137:250921.

47. Head D, Buckner RL, Shimony JS, Williams LE, Akbudak E, Conturo TE, et al. Differential vulnerability of anterior white matter in nondemented aging with minimal acceleration in dementia of the Alzheimer type: evidence from diffusion tensor imaging. Cereb Cortex. 2004;14:410-23.

48. Caffarra P, Ghetti C, Concari L, Venneri A. Differential Patterns of Hypoperfusion in Subtypes of Mild Cognitive Impairment. Open Neuroimag Journal. 2008;2:20-8.

49. Austin BP, Nair VA, Meier TB, Xu G, Rowley HA, Carlsson CM, et al. Effects of Hypoperfusion in Alzheimer's Disease. J Alzheimers Disease. 2011;26(Suppl 3):123-33.

50. Rajani RM, Ratelade J, Domenga-Denier V, Hase Y, Kalimo H, Kalaria RN, et al. Blood brain barrier leakage is not a consistent feature of white matter lesions in CADASIL. Acta Neuropathologica Communications. 2019; 7;187.

51. Liao FF, Lin G, Chen X, Chen L, Zheng W, Raghow R, et al. Endothelial nitric oxide synthase-deficient mice. Am J Pathology. 2021 (In press).

52. Uemura MT, Ihara M, Maki T, Nakagomi T, Kaji S, Uemura K, et al. Pericyte-derived bone morphogenetic protein 4 underlies white matter damage after chronic hypoperfusion. Brain Pathol. 2018;28:521-35.

53. Gladwin MT, Schechter AN, Kim-Shapiro DB, Patel RP, Hogg N, Shiva S, et al. The emerging biology of the nitrite anion. Nat Chem Biology. 2005;1:308-14. 
54. Bryan NS. Natural product chemistry for nitric oxide based therapeutics. Isr J Chemistry. 2019;59:414-9.

55. Endres M, Laufs U, Liao JK, Moskowitz MA. Targeting eNOS for stroke protection. Trends Neuroscience. 2004;27:283-9.

56. Rajani RM, Quick S, Ruigrok SR, Graham D, Harris SE, Verhaaren BFJ, et al. Reversal of endothelial dysfunction reduces white matter vulnerability in cerebral small vessel disease in rats. Sci Transl Medicine. 2018;10:eaam9507.

57. Kirkpatrick PJ, Turner CL, Smith C, Hutchinson P, Murray GD. Simvastatin in aneurysmal subarachnoid haemorrhage (STASH):a multicentre randomised phase 3 trial. Lancet Neurol. 2014;13:666-75.

58. Smith EE, Markus HS. New treatment approaches to modify the course of cerebral small vessel diseases. Stroke. 2020;51:38-46.

59. Kevil CG, Kolluru GK, Pattillo CB, Giordano T. Inorganic nitrite therapy: historical perspective and future directions. Free Radic Biol Medicine. 2011;51:576-93.

60. Elrod JW, Calvert JW, Gundewar S, Bryan NS, Lefer DJ. Nitric oxide promotes distant organ protection: evidence for an endocrine role of nitric oxide. Proc Natl Acad Science USA. 2008;105:11430-5.

61. Tenopoulou M, Doulias PT, Nakamoto K, Berrios K, Zura G, Li C, et al. Oral nitrite restores agedependent phenotypes in eNOS-null mice. JCI Insight. 2018;3:e122156.

\section{Tables}

Due to technical limitations, table 1 to 3 is only available as a download in the Supplemental Files section.

\section{Figures}


A
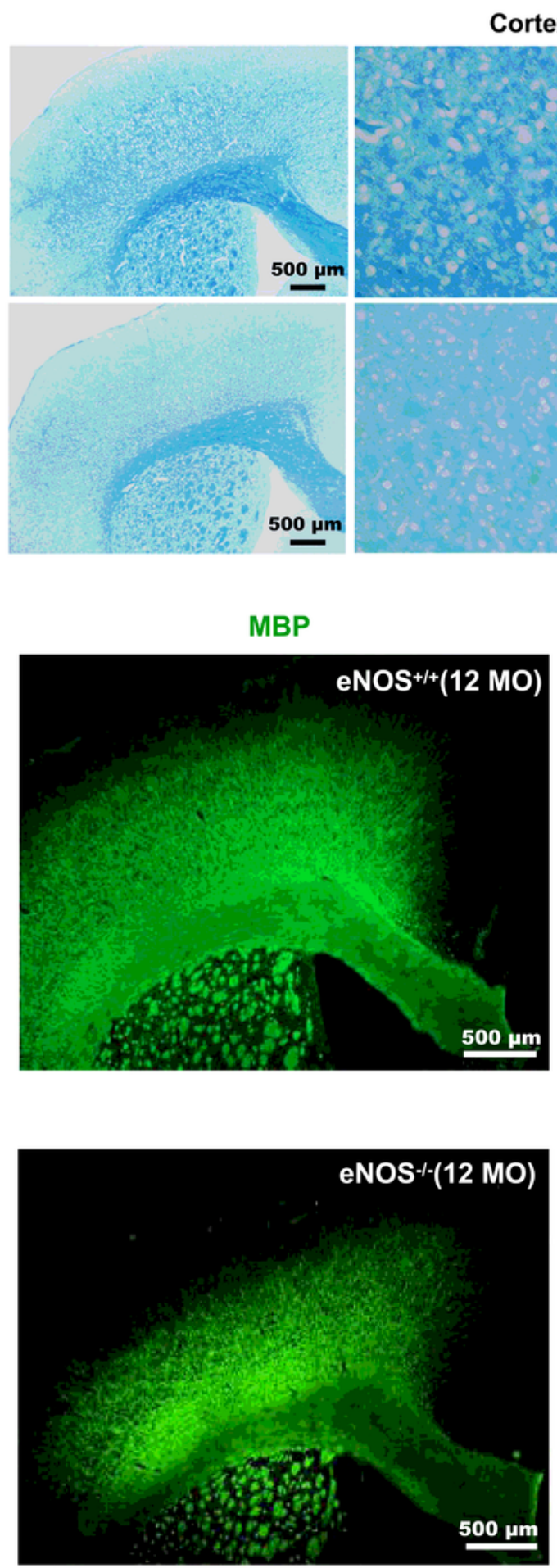

Corpus Callosum

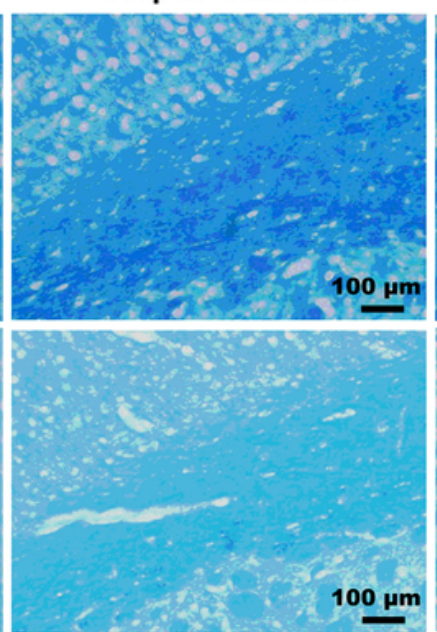

Striatum

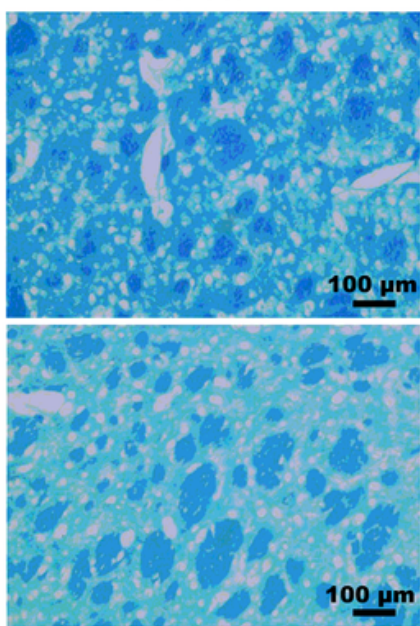

eNOS $^{+/+}$

eNOS $\%$
C

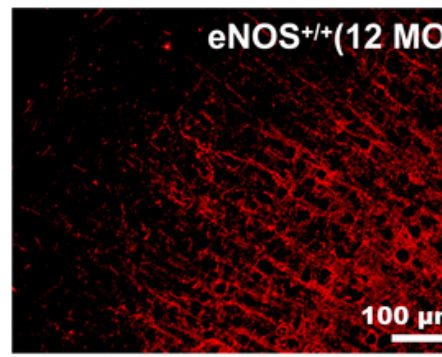

MBP
B
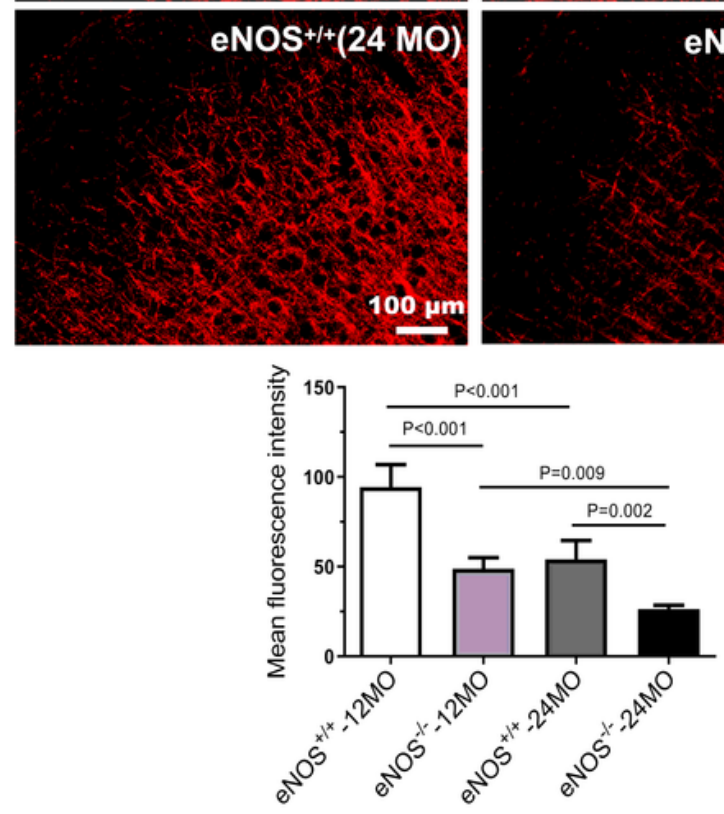

eNOS $-1-(12$ MO)

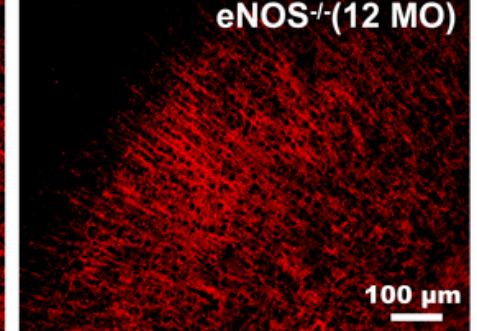

eNOS-1-(24 MO)

\section{Figure 1}

Severe myelin loss in middle-aged eNOS-deficient mice. A) Representative images of LFB (coronal, 16 $\mu \mathrm{m})$ staining taken from mice at 12 months of age. Scaled bars are indicated in each picture. B) Representative images of MBP immunohistochemistry at 12 months of age. Scaled bars: $500 \mu \mathrm{m}$. C) Representative images of MBP immunohistochemistry from the frontal cortical regions at 12 months of age. Scaled bars: $100 \mu \mathrm{m}$. Quantification was performed based on the mean fluorescent intensity from the frontal cortical regions and expressed as mean \pm s.e. $m ; \mathrm{N}=5-6$ mice/group. 
A

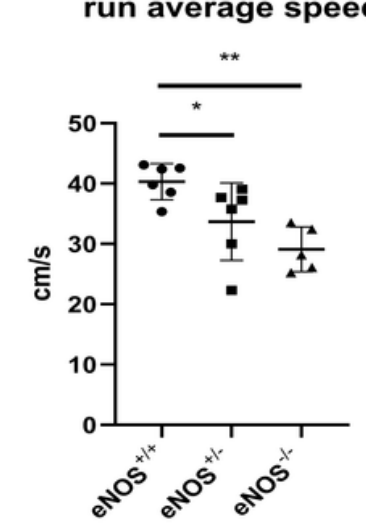

run max variation

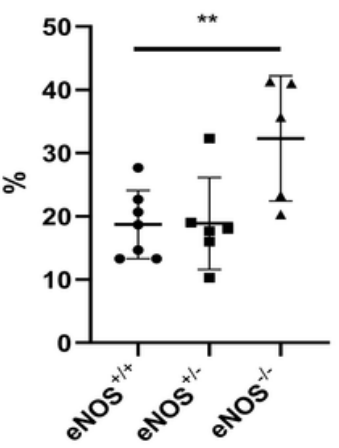

B

LFB+NissI outer pyramidal layer (III) inner pyramidal layer (V)
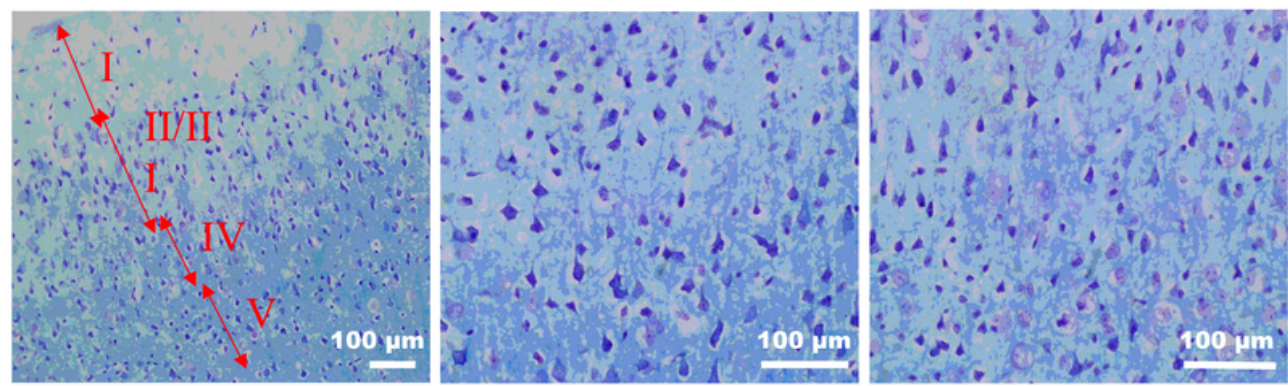

eNOS $^{+/+}$
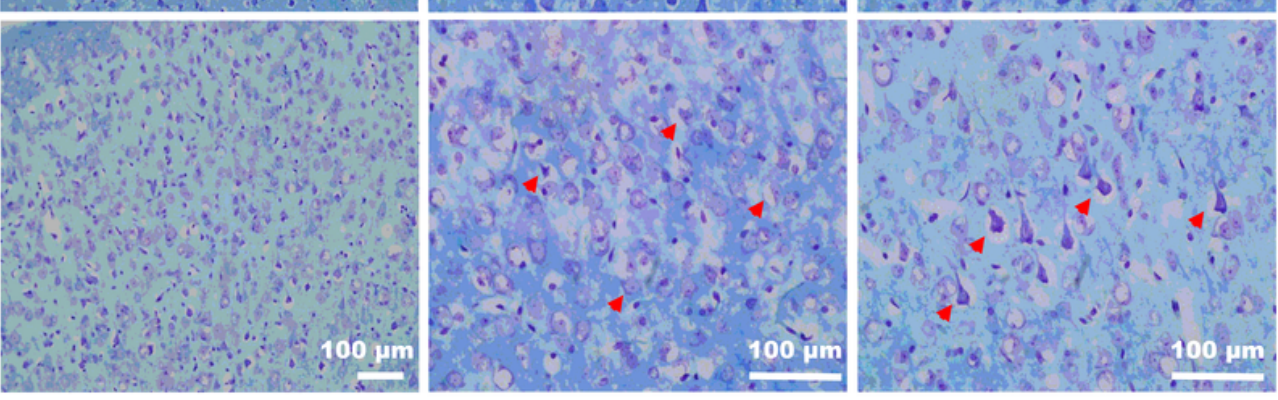

eNOS -

Figure 2

Gait disbalance and cortical pyramidal neurodegeneration. A) Gait performance by CatWalk testing. The test started once the mouse entered the visual field of the camera, and stopped once the mouse disappeared. The averaged running speed and additional parameters (e.g., altered sequence patterns and inter-limb coordination) were recorded, analyzed and expressed as mean \pm s.e.m; $\mathrm{N}=13-15$ mice/group per genotype. ${ }^{*} \mathrm{P}<0.05$ and ${ }^{*} \mathrm{P}<00.005$. B) Representative images of LFB and Nissl double-stained sections of brains of eNOS-/- and control littermates showing frontal cortical regions. Red double arrows and the corresponding Roman numerals indicate cortical layers I through V. Red arrowheads indicate degenerating neuron with swollen soma. Scaled bars are indicated in each picture. 
A
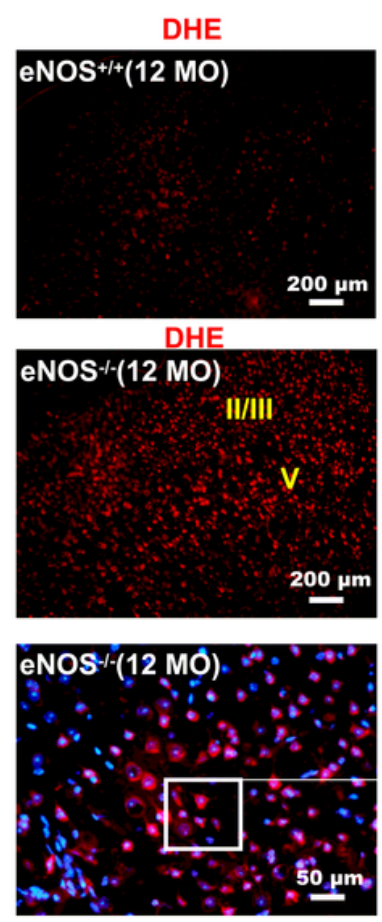

\section{B}
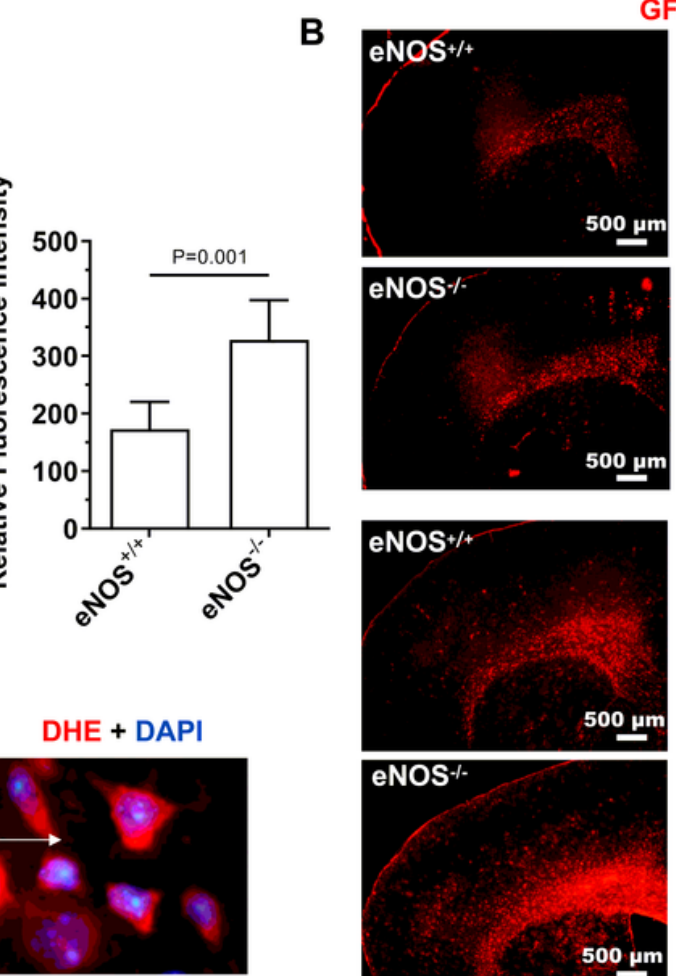

GFAP
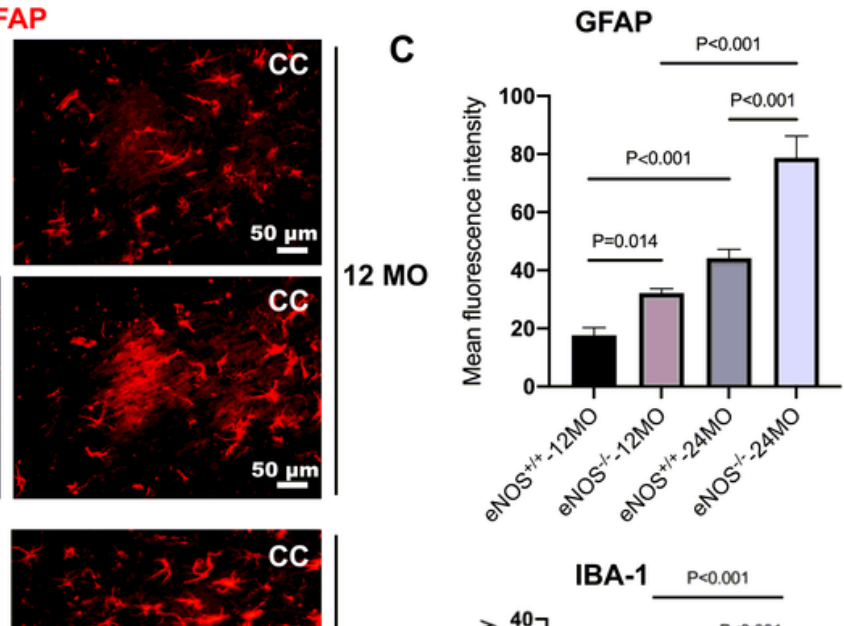

IBA-1 P<0.001

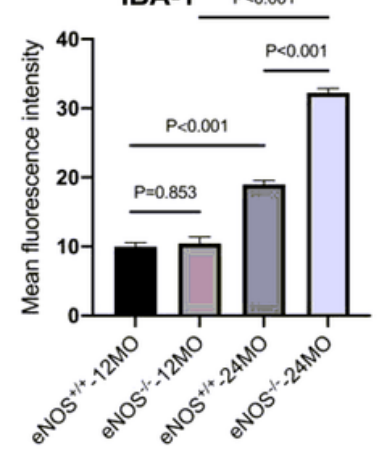

\section{Figure 3}

Elevated reactive oxygen species (ROS) and astrogliosis. A) Representative images of dihydroethidium (DHE)-stained frontal forebrain cortical regions from mice at 12 months of age. Roman numerals in yellow indicate cortical layers II/III and V where we detected most elevated ROS signals. Lower right panel images with zoned area showing intracellular DHE signals presumably deriving from the clustered mitochondria in cytoplasm. Quantification was performed based on the mean fluorescent intensity from the frontal cortical regions and expressed as mean \pm s.e.m; $N=5-6$ mice/group. B) Representative images of GFAP immunohistochemistry on coronal brain sections of mice at 12 and 24 months of age. C) Quantification of GFAP and Iba-1 immunofluorescence intensity based on 5-6 mice/group and expressed as mean \pm s.e.m. 
A

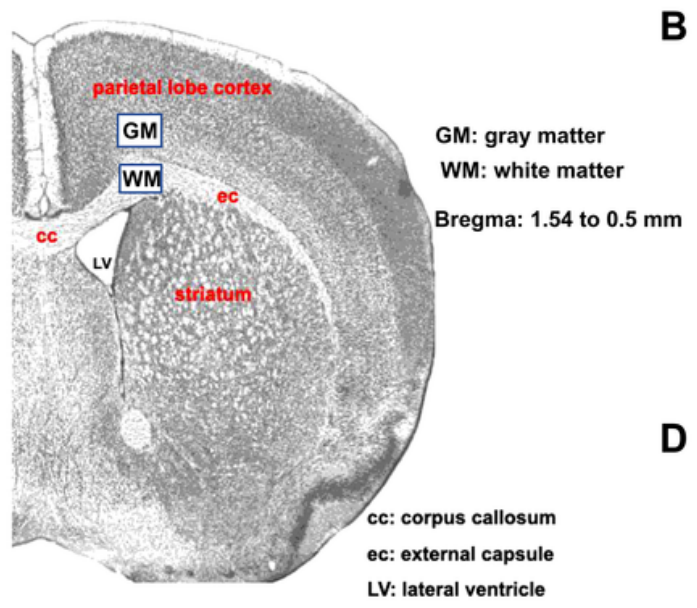

C

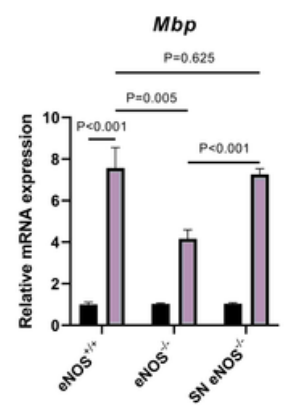

B

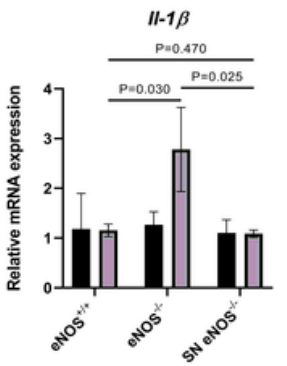

D
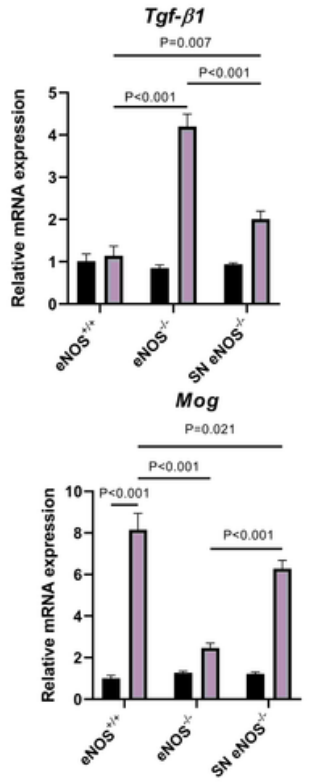
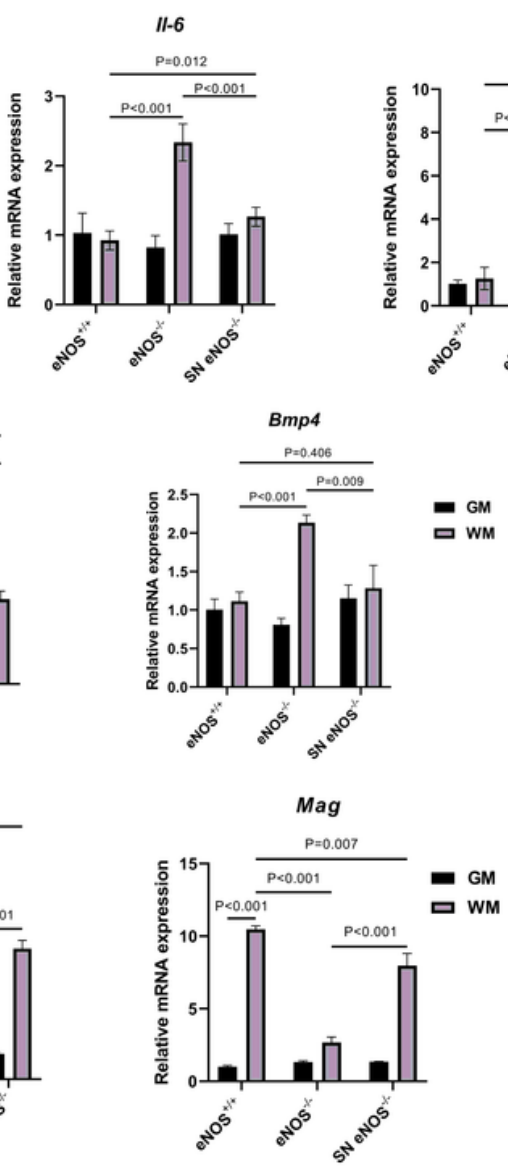

Figure 4

Gene expressional changes in white matter tissue as determined by quantitative qPCR. A) Diagram of the specific brain region tissue for micro punch. $B$ ) hree representative genes encoding for pro-inflammatory cytokines. II-1 $\beta$ : interleukine-1 $\beta$; II-6: inetrleukine-6; Tnf-a:tumor necrotic factor- $\alpha$. C) myelin-pathway genes: Mbp: myelin binding protein; Plp: myelin proteolipid protein or lipophilin; Mog: myelin oligodendrocyte glycoprotein; Mag: myelin associated glycoprotein. D) Two genes of the transforming growth factor TGFa family. Tgf-1 $\beta$ : transforming growth factor-1 $\beta$; Bmp4: bone morphogenetic protein 4 . Data are presented as mean \pm s.d.m., normalized by gapdh gene expression. $\mathrm{N}=3-4$ mice/group. 
A
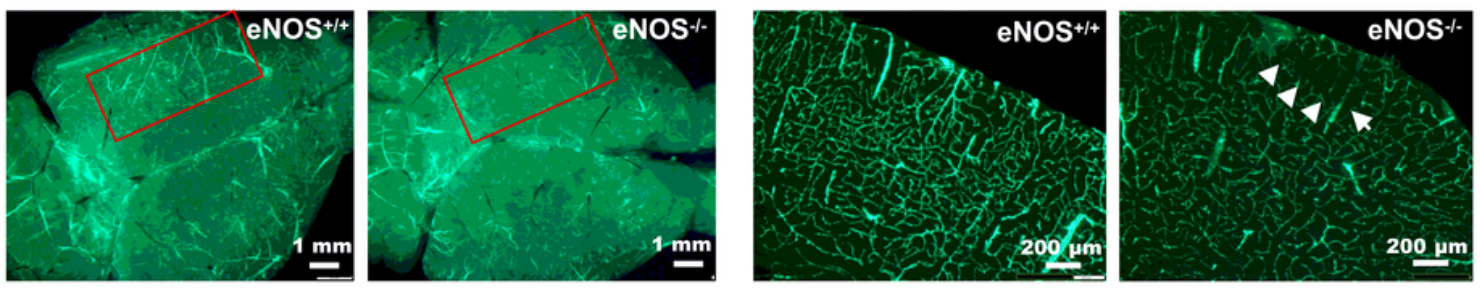

FITC-dextran

(2000 kDa)

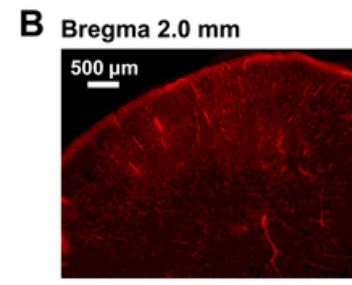

Bregma $1.42 \mathrm{~mm}$

Bregma $0.26 \mathrm{~mm}$

Bregma $-0.22 \mathrm{~mm}$

Bregma $-1.82 \mathrm{~mm}$
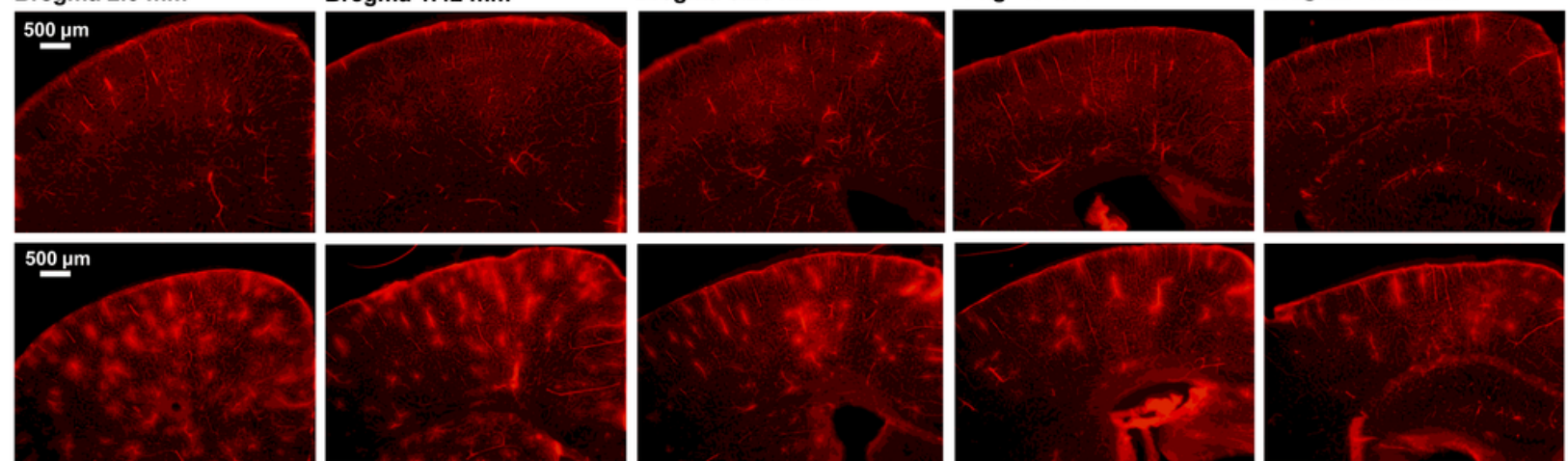

eNOS $+/+$
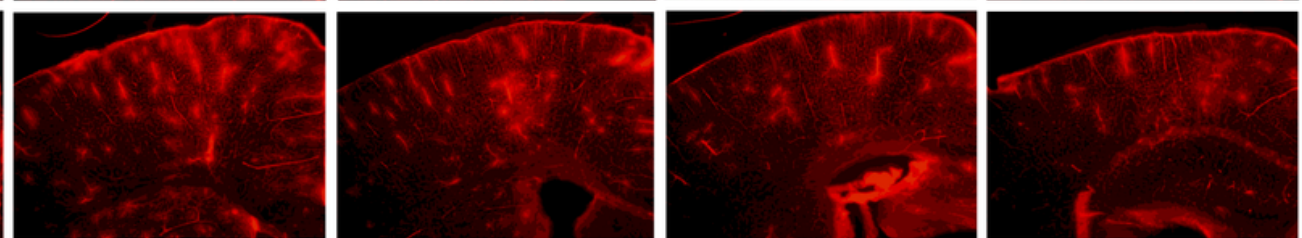

eNOS $*$
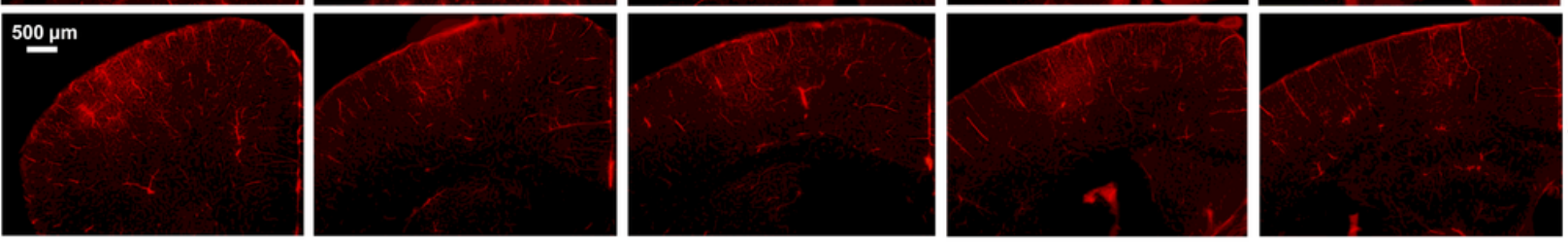

\section{Figure 5}

Cerebral FITC-dextran and Evans blue angiography. A) Representative FITC-dextran angiograms of whole brain (left two panels) from dorsal view from eNOS mice at 3 months of age. Red boxed indicates parietal cortical zone from dorsal view. Right two panels show the representative FITC angiograms taken from $100 \mu \mathrm{m}$ coronal sections of frontal brains from the same mice as in the left two panels. B) Representative images of Evans blue angiograms of young eNOS $+/+$ and eNOS-/- mice (3 months of age) taken at 2.0 to - $1.82 \mathrm{~mm}$ Bregman, showing BBB leakage detected at young age which can be prevented by sodium nitrate (SN) feeding in drinking water for 6 weeks starting from 8-week-old (SN eNOS-/-, N=3 mice/groups). Evans Blue ( $2 \%$ in sterile water) was injected in $150 \mu$ volume through mouse tail vein. Mice were euthanized $5 \mathrm{~min}$ later without perfusion and whole brains removed and vibratome-processed to $100 \mu \mathrm{m}$ serial sections for fluorescent imaging using an rhodamine filter. Scaled bars: $500 \mu \mathrm{m}$. 
A

Sodium Nitrate (SN) Feeding

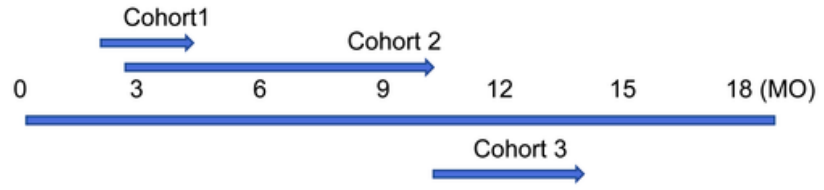

B

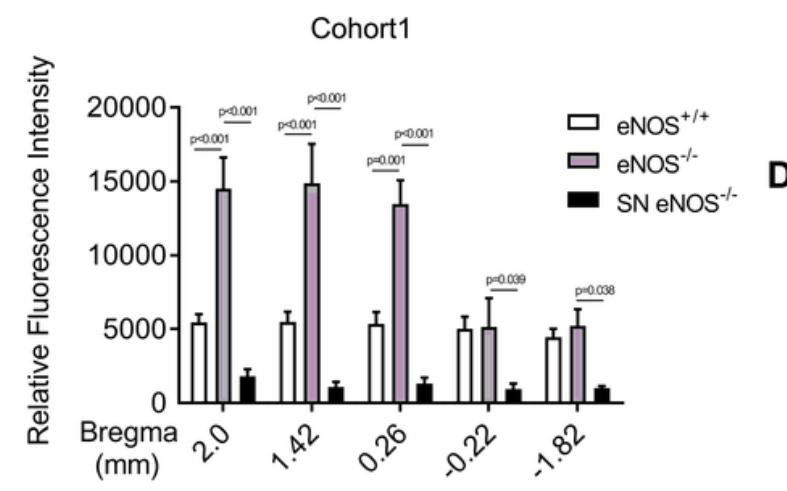

Cohort 2
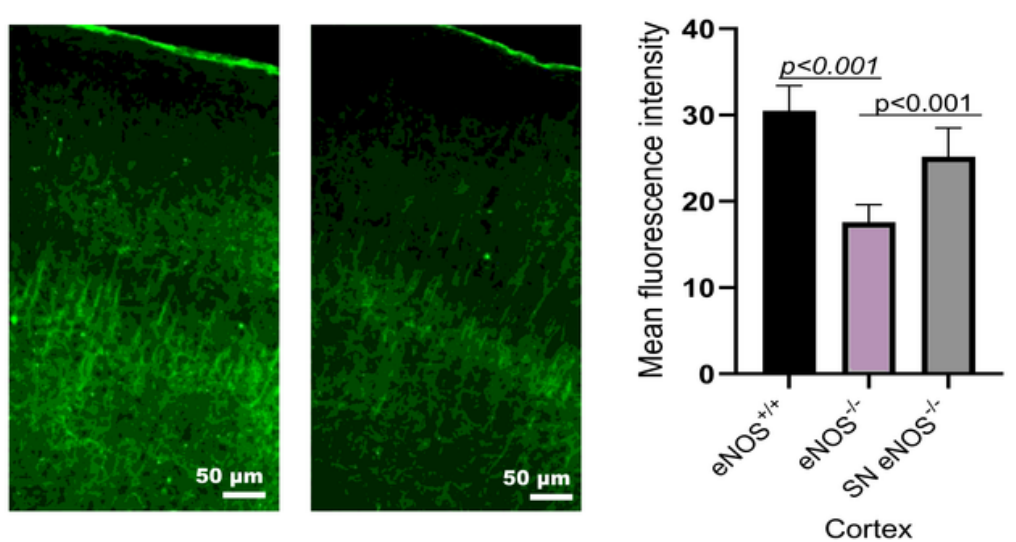

Cohort 3
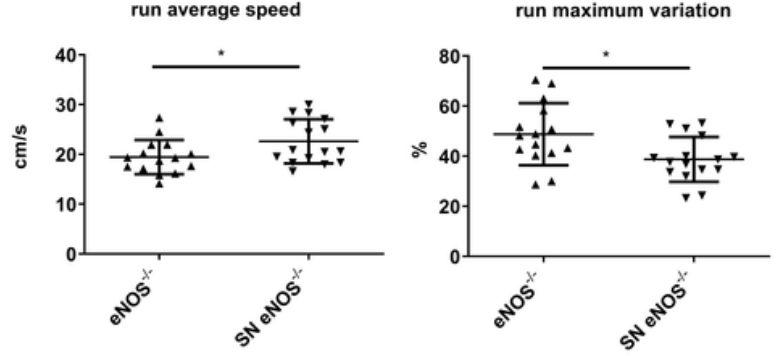

Figure 6

Restoring NO signaling by sodium nitrate feeding rescues white matter pathology and functional phenotypes. A) Schematic diagram illustrating sodium nitrate (SN) feeding schedules in three cohorts of mice. B) Quantification of Evans blue fluorescent signals based on the angiograms presented in Figure 5B. Data are presented as mean \pm s.e.m based on $\mathrm{N}=3$ mice each group. C) Representative images of SMI-32 immunohistochemistry (Cohort 2 mice). D) Gait performance in post-symptomatic eNOS-/- mice with and without SN feeding for 4 months (Cohort 3 mice); data are presented as mean \pm s.e.m., $N=17-19$ mice/group. * indicates $\mathrm{P}<0.05$.

\section{Supplementary Files}

This is a list of supplementary files associated with this preprint. Click to download.

- SupplementaryFigure1.tif

- SupplementaryFigure2.tif

- SupplementaryFigure3.tif

- SupplementaryFigure4new.tif

- SupplementaryFigure5.tif

- SupplementaryFigure6.tif

- Table1.png

- Table2.png 
- Table3.png

Page 29/29 